\title{
VINCENT Visualization of Network Centralities
}

\author{
Andreas Kerren, Harald Köstinger, and Björn Zimmer \\ Linnaeus University, School of Computer Science, Physics and Mathematics (DFM), ISOVIS Group, \\ Vejdes Plats 7, 35195 Växjö, Sweden \\ Corresponding author: andreas.kerren@lnu.se
}

Keywords: Centralities, Network Analysis, Visualization, Biological Networks, Graph Drawing, Coordinated Views.

Abstract: The use of network centralities in the field of network analysis plays an important role when the relative importance of nodes within the network topology should be rated. A single network can easily be represented by the use of standard graph drawing algorithms, but not only the exploration of one centrality might be important: the comparison of two or more of them is often crucial for a better understanding. When visualizing the comparison of several network centralities, we are facing new problems of how to show them in a meaningful way. For instance, we want to be able to track all the changes of centralities in the networks as well as to display the single networks as best as possible. In the life sciences, centrality measures help scientists to understand the underlying biological processes and have been successfully applied to different biological networks. The aim of this paper is to present a novel system for the interactive visualization of biochemical networks and its centralities. Researchers can focus on the exploration of the centrality values including the network structure without dealing with visual clutter or occlusions of nodes. Simultaneously, filtering based on statistical data concerning the network elements and centrality values supports this.

\section{INTRODUCTION}

In Information Visualization or Graph Drawing, there are many different approaches to visualize complex networks that support various exploration methods. Such networks (or graphs) can be drawn, for example, by using standard graph drawing algorithms [Di Battista et al., 1999, Jia et al., 2008]. In case of social networks and their visual analysis based on any graph representation, important tasks are the identification of communities and central actors as well as the analysis of roles and positions [Henry et al., 2007]. Those tasks allow researchers to find the most relevant parts and correlations in social networks.

Another example are biochemical networks, which are representations of biological processes, such as metabolism, the regulation of genes, or the interaction of proteins. They have been of strong interest in the last few years and are crucial for a comprehensive understanding of living beings [Jusufi et al., 2011, Albrecht et al., 2010]. In this paper, we focus on this application field. In the life sciences, centrality measures help scientists to understand the underlying biological processes and have been successfully applied to different biological networks. Network cen- trality analysis measures the relative importance of nodes in a network based on their connectivity within the network structure [Dwyer et al., 2006, Koschützki and Schreiber, 2004]. Applications in biological networks can be found at the investigation of proteinprotein interaction networks (PPI) or transcriptional regulatory networks (TR) [Koschützki and Schreiber, 2004]. Typical tasks for such network analysis by the use of centrality values are: (a) finding nodes with high centrality values, since those are more likely of interest to the researcher; (b) finding nodes with low centrality values to hide them, since they are of less importance; (c) finding nodes with high values in several centralities (comparisons of values over many nodes). Especially the latter task is challenging, because important problems arise when visualizing it. For example: How to visualize the data in a way that researchers can get the most meaning out of it? How enabling the user to keep track of centrality changes within the network? How to minimize occlusions and visual clutter? Or how to build a flexible solution in order to deal with a large number of centrality values at the same time?

The aim of our work is to overcome the aforementioned problems and to develop a new solution of vi- 
sualizing networks together with its centralities. We introduce a new visual representation of networks and their centrality values in a circular view. Analyses can then be focused on the exploration of the centrality values including the network structure, without dealing with visual clutter or occlusions of nodes. Filtering based on statistical data concerning the network elements and centrality values supports this and helps keeping the network itself readable. Hereby, the comparability of the nodes is one of most important goals to fulfill, followed by the minimization of visual clutter and occlusions.

The remainder of this paper is organized as follows. The next two sections provide a brief overview of network analysis and network centrality concepts and motivate their importance for visual network analysis on the basis of biological networks. Related work and actual challenges of the visual analysis of network centralities are summarized too. To address those problems, our tool ViNCent is introduced in Section 4. Here, the system and its design are explained in detail. An explicit description of the methods and approaches used to solve the described problems is given too. Section 5 exemplifies the interaction design of our tool based on a small use case scenario. A discussion at the end of this section summarizes advantages and disadvantages of our tool. The conclusion and future work section deals with possible improvements of the tool and planed further work.

\section{BACKGROUND}

This section provides additional background information to facilitate the understanding of the rest of the paper. First, a brief introduction into graphs is given including the most important definitions.

A graph provides information about single elements and relationships between those. A (simple) graph $G=(V, E)$ consists of a finite set of vertices (or nodes) $V$ and a set of edges $E \subseteq\{(u, v) \mid u, v \in$ $V, u \neq v\}$. An edge $e=(u, v)$ in the graph $G$ connects two nodes $u$ and $v$. Two nodes $u$ and $v$ are said to be incident with the edge $e=(u, v)$ and adjacent to each other. The degree $d(u)$ of a node $u$ is defined as the number of edges incident to this node $u$. Furthermore, we can define a walk on a graph as described as follows: let $\left(e_{1}, \ldots, e_{k}\right)$ be a sequence of edges in a graph $G=(V, E)$. This sequence is called a walk if there are nodes $v_{0}, \ldots, v_{k}$ such that $e_{i}=\left(v_{i-1}, v_{i}\right)$ for $i=1, \ldots, k$. If the edges $e_{i}$ and the nodes $v_{i}$ are pairwise distinct respectively, then the walk is called a path. The length of a walk/path is given by its number of edges, i.e., $k=\left|\left(e_{1}, \ldots, e_{k}\right)\right|$. A shortest path between two nodes $u, v$ is a path with minimal length. The distance (dist $(u, v)$ ) between two nodes $u, v$ is the length of a shortest path between them. [Jusufi et al., 2010, Görg et al., 2007, Di Battista et al., 1999]

\subsection{Network Analysis}

In the sciences, huge networks are used to model structural relationships of various types. Therefore, network analyses for social, biological or computer sciences become more important as well to support a better understanding of the underlying network structures [Newman, 2010]. The following paragraphs deal with network analysis and identify important tasks by means of biological networks.

Analysis of Biological Networks Junker et al. state in their work: "structural analysis of networks can lead to new insights into biological systems and is a helpful method for proposing new hypotheses" [Junker et al., 2006]. For structural analysis, several techniques exist: analysis of the global network structure, network motifs (i.e., small subnetworks, which occur more often within the whole network), network clustering, and network centralities. The latter technique uses far more information about the network than just the relationships and neighborhood of nodes. In fact, this technique uses centralities of nodes to rank the elements in the network according to a given importance concept [Junker et al., 2006]. The following Subsection 2.2 discusses network centralities in general and how they are calculated. A presentation of suitable visualization techniques follows in Section 3.

\subsection{Network Centralities}

A network centrality $C$ is a function that assigns a value $C(u)$ to a node $u \in V$ of a given graph $G=(V, E)$. In order to compare network centralities according to their importance, $u$ is more important than $v$ iff $C(u)>C(v)$ [Koschützki and Schreiber, 2004, Dwyer et al., 2006]. Network centralities are used for a better understanding of complex processes in networks. In the life sciences, centrality measures are useful to understand biological processes. They are therefore applied to biological networks and then explored. The following two sample centralities are typically used by scientists to receive further meaning of networks [Junker et al., 2006, Dwyer et al., 2006, Koschützki and Schreiber, 2004]:

Eccentricity $C_{e c c}$ : This network centrality is calculated by using the distance between nodes in the graph. The eccentricity ecc of a node $u$ is defined 
as ecc $(u):=\max _{u \in V} \operatorname{dist}(u, v)$ and the corresponding centrality as $C_{e c c}(u):=\frac{1}{e c c(u)}$. More central nodes have therefore a higher value of $C_{e c c}$.

Random Walk Betweenness $C_{r}$ : Betweenness centralities model communication paths in networks and measure the extent to which a node lies on paths between other nodes. For $C_{r}$, the centrality of a vertex $w$ is equal to the number of times that a random walk from $u$ to $v$ goes through $w$, averaged over all $u$ and $v$ [Newman, 2003].

The actual calculation of centralities is not complex. Even for large-scale networks, this can be done very fast and be cached to speed up a later interactive exploration. The complexities of the individual centralities range from $O(n)$ to $O\left((n+m) n^{2}\right)$ [Koschützki and Schreiber, 2004].

The problem of choosing the right centralities differs from network to network. For the computation of suitable centralities, data about the functional properties of networks is often missing. This data would allow to choose the "right" centrality measures, which show the important parts of the network. Therefore, this analysis is usually done by visually comparing the centrality values on the networks [Dwyer et al., 2006].

\section{RELATED WORK}

In this section, we provide a short overview of related work in context of the visualization of network centralities. Additionally, we outline the most important challenges. Because of space limitations, we restrict ourselves to a brief presentation of tools in biochemical network visualization and refer to the survey paper [Albrecht et al., 2010] and the book [Junker and Schreiber, 2008]. For the field of social networks, we refer to the work [Correa and Ma, 2011].

The visualization of network centralities was not much discussed in the literature so far. Typical methods, as stated by Dwyer et al. [Dwyer et al., 2006], are the use of correlations, scatter plots, and parallel coordinates. The problem with these solutions is, that they have disadvantages when used for biological networks, since correlations of centralities might occur anyway. The most important issue is not only to show that there are correlations, but to show where those correlations occur within the network. In their work, Dwyer et al. present three new techniques to visualize network centralities as described in the following [Dwyer et al., 2006].
3D Parallel Coordinates-based Comparison: This method is based on parallel coordinates to visualize multivariate data. Standard approaches typically deal with two dimensions. This one uses 3D to stack visual representations of a network according to one centrality into the third dimension. Thus, each 2D plane contains the information for a particular centrality. 3D Parallel Coordinates-based Comparison gives a good overview of the centrality values within the network and about how many nodes fall into a certain value range [Dwyer et al., 2006]. However, this approach does not reveal the actual network structure.

Orbit-based Comparison: Arranging nodes in an orbit-based visualization has some advantages over the previous approach: the network topology is shown and thus the relationships between the nodes can be identified. In more detail, for each centrality a new $2 \mathrm{D}$ orbit-based plane is added to the $3 \mathrm{D}$ drawing. The ordering of the planes takes the edge crossing minimization and the minimization of inter-plane edge lengths into account [Dwyer et al., 2006]. As a single orbit provides information about the centrality values and as the network structure can be seen, this approach outperforms the previous one when revealing both structure and centrality values. Drawbacks are occlusions in the middle of the orbits, and it is hard to keep track of changes within the single centrality measures.

Hierarchy-based Comparison: This approach is conceptually similar compared to the 3D parallel coordinates approach, but it divides the nodes according to their centrality values into layers. Those layers are then drawn as horizontal lines, having an ordering on the line as well. This could be, for example, a decreasing centrality value from the left to the right. The top layers in the visualization are considered to show larger centrality values. There might be even connecting edges between nodes on the same layer. Filtering and thresholds are used to reduce visual clutter between two planes [Dwyer et al., 2006].

CentiBiN: Junker et al. present a different approach with the CentiBiN tool [Junker et al., 2006]. CentiBiN uses standard node-link diagrams based on a set of graph drawing algorithms. In addition to the displayed graph, single centrality values are displayed next to the visualization in a table. Interactions with the table, like selecting certain centrality values, are coordinated with the network visualization as well. So, it allows the user to locate certain values within the network. Simple histograms are used to compare 
data. CentiBiN has advantages with respect to the amount of available centralities, as it can calculate up to 17 centrality measures for networks.

CentiScaPe: This tool is able to compute several network centralities and provides analyses of existing relationships between user data (based on experiments) and centrality values computed by CentiScaPe itself [Scardoni et al., 2009]. It was implemented as Cytoscape plugin and supports even large input networks. However, the supported interactive visualizations are restricted.

Challenges All aforementioned tools and approaches solve the problem of network analysis according to centrality values. But there is still space for improvements, such as a better arrangement of nodes and planes, avoiding occlusions and visual clutter, visualizing structure and centralities simultaneously, or introducing new interaction and filter techniques.

\section{VINCENT}

ViNCent-short for Visualization of Network Centralities - solves most of the problems addressed in the previous Subsection 3 by using a radial graph drawing approach [Kerren and Köstinger, 2011]. Each network node is represented by a small quadrangle that is positioned on a circle. Its connections to the other nodes (i.e., the edges) are laid out inside of this circle. Figure 1 shows an example of such a radial layout in its center: it is easy to see how nodes are interconnected and how many connections a node has. Features like edge bundling and degree marking, as described later in Section 4.2, support the user in finding important relationships between nodes as well as highly or lowly connected nodes.

Before we describe single features of our tool, we give an overview of the overall design and architecture. ViNCent provides multiple, coordinated views on the input data (mainly by using the Prefuse toolkit [Heer et al., 2005]), see Figure 1. They are briefly discussed in the following.

Settings Panel The user can change the visual appearance of the tool, generate random network and centrality data for testing purposes, etc. via the controls in the settings panel.

Circle View The circular network drawing in the center displays the nodes, their centrality values, and the graph structure itself. Therefore, this view provides an overview of the complexity of the entire network and supports the user to get the main actors at a first glance. Our tool offers two possible layouts of a node's centrality representation (called centrality bar in this paper): traditional stacked bars and maximum value stacking. Whereas for traditional stacking the single bars corresponding to centrality values are immediately stacked onto each other, the maximum value stacking starts all bars from the level of the maximum value of the current centrality, thus providing a better comparability of relative centrality values. Figure 1 shows the differences between the two modes.

Histogram View One individual histogram provides a statistical overview of a centrality's values. Thus, the histograms help the user to better understand the distributions of centrality values over all nodes.

Centrality Hover View The single centrality bars are not only displayed in the circle view; a selected bar is redundantly visualized together with detailed information about the corresponding node's centrality values, i.e., centrality name, relative percentage, and absolute value. Figure 1 shows this view displaying data of the currently hovered node 44 in the lower right area of the main window.

\subsection{Interaction-Concepts in ViNCent}

The aforementioned views provide the user with the possibility of doing further interactions, such as hovering bars and filtering out data based on the distribution of centrality values as well as the network topology.

\subsubsection{Linking and Brushing}

ViNCent makes extensive use of linking and brushing features [Keim, 2002, Roberts, 2007] to connect certain data objects in the visualization. Hovering features are introduced to highlight elements and show cross-connections:

Hovering in the Circle View The main focus of the user is usually on the circle view in the center, cf. Figure 1. Hovering nodes in this window leads to an activation of the connected nodes (their neighborhood), the connecting edges, and the corresponding labels. By the use of the settings panel, the user can control how many hierarchy levels in the network the highlighting spreads out in the view. This feature of highlight spreading highlights nodes and corresponding edges weaker and weaker depending on the hierarchy level. 


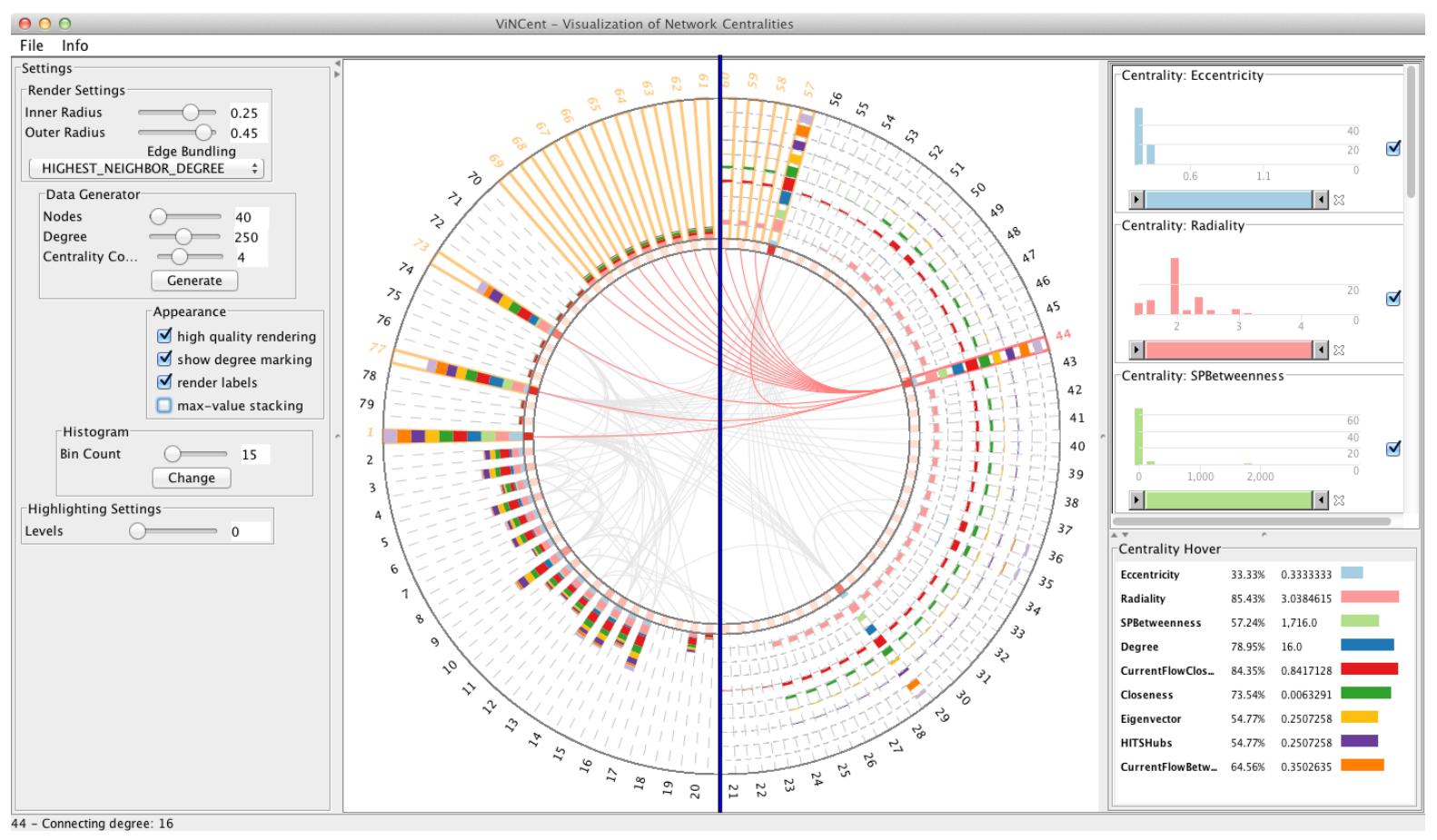

Figure 1: Overview of the ViNCent tool. The center view shows the radial drawing of a network. The two possible drawing modes (normal stacked bars (left half) and maximum value stacking (right half)) are shown as overlay (split by the blue line). To the right, the corresponding histograms of the network centralities are shown (top) as well as more detailed information about the currently selected node 44 (bottom). Histograms can be used to filter the views. To the left, the settings panel allows the user to change tool parameters and to generate data sets for testing purposes.

Hovering in the Histograms View To show the user which nodes fall into which certain range of data values according to one centrality measure, hovering an individual histogram bar highlights the corresponding nodes in the circle view as well. Thus, the user can check in more detail, which nodes are related to a histogram bin and would be affected by filtering them out.

\subsubsection{Filtering and Dynamic Queries}

The exploration of big datasets is mostly not possible without filtering of data and dynamic queries [Shneiderman, 1994]. ViNCent uses several approaches to allow the user to filter out data and therefore to reduce the dataset to a smaller amount of nodes. In this way, filters support the user in fulfilling his/her tasks more efficiently. Since the centrality data correlated to the network is multidimensional, we decided to use an approach that was originally realized by Attribute Explorer [Tweedie et al., 1994]. It maps each node attribute (i.e., its centrality values) to a histogram.

Filtering processes in ViNCent are based on the distribution of centrality values over the nodes. In our case, filtering out nodes means filtering out the corre- sponding edges in the circular view as well in order to clear the center of the visualization up and to reduce the visual clutter. Hereby, ViNCent supports four different filter options:

Filtering based on histogram bars The first possibility is to filter out elements belonging to a specific bar of a histogram. By clicking the bar, the corresponding elements in the circle view are hidden. The bar in the histogram is therefore marked in light gray color, which symbolizes that this bar has been filtered out, see Figure 2(c).

Filtering based on histogram sliders Another way of hiding elements is to apply the range sliders below each histogram. Figure 1 (right) shows examples of range sliders for several centralities. By sliding from the left or right, the amount of displayed nodes decreases corresponding to the eliminated elements in the dataset. This method is useful to quickly filter out minimum and maximum values of histograms.

Filtering based on single nodes As the filters presented before act on the whole set, they affect more nodes at once. But to filter out single nodes from the view, our penultimate filter option can be useful, which is based on single nodes. A right- 


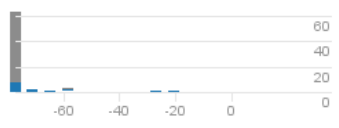

(a) 'Centroid' $C_{c e n}$

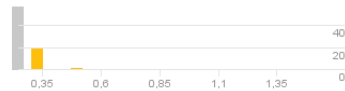

(c) 'Eccentricity' $C_{e c c}$

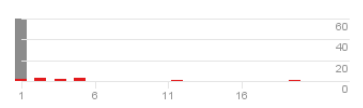

(b) 'Degree' $C_{d e g}$

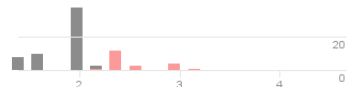

(d) 'Radiality' $C_{\text {rad }}$
Figure 2: Histograms for four different centralities. Figure 2(c) has an active filter on the first bar, shown in light gray. This active filter spreads to the other histograms, displayed in dark gray.

click on nodes in the circle view causes that they disappear from the view. Renewed right-clicking brings them up again.

\section{Hiding centralities from the circle view}

Sometimes, certain centrality values-and therefore the whole centrality-have no further meaning for the exploration of the dataset. For instance, if all nodes have the same centrality value. In this case, the centrality can be hidden by deselection of the corresponding visibility check box, see Figure 1 (right).

Filter Propagation Filtering out elements affects directly the circle view; Figure 3 shows the results of filtering processes performed by the user. Thus, direct feedback based on such actions is provided to the user. But to better keep track of already eliminated elements, they are marked in the histograms as light gray bars, when the filter is applied directly on this histogram. The bars are marked in dark gray, when the filter spreads from another active filter in a different histogram. This filter propagation gives the user a more precise feedback of how certain centrality values are related to each other, cp. Figure 2.

\subsection{Implementation Aspects}

The advantages of our tool are its capabilities concerning the interactive exploration of the nodes and the display of a number of different centrality values at the same time. These features are mainly achieved by the circular arrangement of nodes and the additional edge bundling approaches done in the middle of the circle view. The latter support the user in finding connections between nodes and showing if nodes are highly or lowly connected. In the following, we discuss the most important technical aspects of these features.

For the circular arrangement of the nodes, the entire available space in the view is taken for drawing

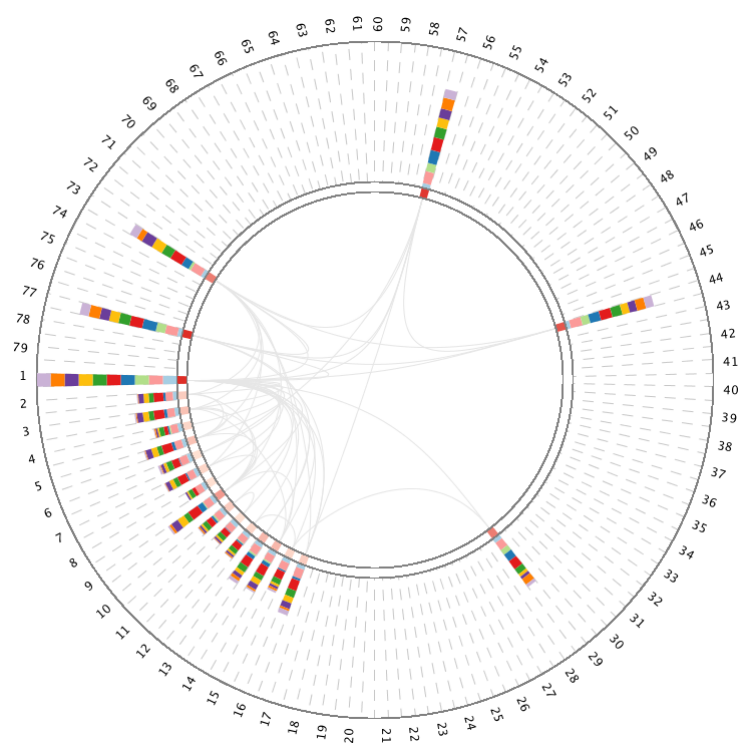

Figure 3: Active filter on the network. Nodes have been filtered out by using different filter options of the histograms. The active eccentricity histogram bar filter eliminated all nodes having a low value in this centrality. As Figure 2(c) shows, about 55 nodes are affected by this active filter.

the inner circular disk and the outer circular ring. Between both, the nodes are added and represented as small squares. Their degree is represented by a color gradient from light-red to dark-red (connection degree marker) and their centrality values as bar drawings on the outer ring. The arrangement is done by simply dividing the whole $360^{\circ}$ circle into single-angle steps, used to define the positions of the nodes. In order to distinguish between single centrality values, a specific color schema is employed. ViNCent uses a color schema provided by ColorBrewer [Brewer, 3 $22]$. Thus, a centrality gets assigned a specific color from this schema which is consistently used in all views.

Graph drawing techniques usually have to deal with occlusions and visual clutter when it comes to more dense graphs. This is prevalently the case in the area of network analysis, especially for biological networks. In order to reduce visual complexity in our views, the circular arrangement of nodes avoids occlusions of them. However, there is still the problem of visual clutter in the center of the circle view where the connecting edges are located. One technique to solve this problem is hierarchical edge bundling [Holten, 2006]. It basically follows a simple principle: visually bundling adjacency edges together analogous to the way electrical wires and/or network cables are merged into bundles along their joint path. ViNCent deals with graphs as well, but our tool draws all nodes to the outside of the circle. Therefore, no hi- 
erarchy can be used in its center to perform standard edge bundling based on the inner hierarchy. Instead, ViNCent supports four different edge bundling modes that are explicitly described in the thesis [Köstinger, 2011]. Similar approaches exist in the literature, such as those described in [Correa et al., 2008].

\subsubsection{Plug-in: CentiBiN}

ViNCent is actually not limited to a certain field of application. It can handle every network, if it is represented in GraphML container format. Our tool also accepts precomputed (numeric) centrality values that are stored in the input file as additional attributes. Then, ViNCent can directly visualize the multivariate network without any preprocessing steps. An excerpt from such an extended GraphML file is given in the following:

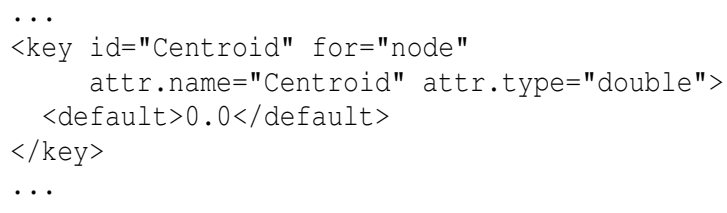

To support more application domains-especially biological network analysis-ViNCent uses the CentiBiN plug-in [Junker et al., 2006] that calculates up to 17 network centralities for biological networks. After loading the input graph in GraphML format, the CentiBiN plug-in can calculate a user-specified number of centralities on directed and undirected graphs, e.g., degree, eccentricity, etc. Then, ViNCent visualizes the input graph together with the computed centrality values. Additionally, the user can export all data into an extended GraphML file. Thus, users are able to reload the network together with its centrality values without recalculation. Note that not every centrality measure can be applied to every graph, since there are preconditions a graph has to fulfill in order to calculate the values. These can be simplicity, connectedness and loop-freeness [Junker et al., 2006]. One example is the Eigenvector-centrality whose implementation in CentiBiN requires that the input graph has to be loop-free.

\section{USE-CASE SCENARIO AND DISCUSSION}

Before we discuss the pros and cons of ViNCent, a short use-case scenario shows how the tool can be used for biological network analysis. It is described in the following section.

\subsection{Use-Case Scenario}

We use the release 2005-01-26 of the Mus mиsculus dataset from the Database of Interacting Proteins (DIP) [Salwinski et al., 2004] as an example for investigating biological networks. It describes proteinprotein interactions (PPI) of the house mouse and consists of 49 nodes and 54 edges. The aim is to find the most important proteins by visualizing their network centralities with our tool.

Figure 4(a) shows the selected dataset for this usecase. Protein $320 \mathrm{~N}$, which has the highest overall centrality values, is selected and its adjacent proteins are highlighted. Detailed centrality numbers for the selected protein are displayed in the hover panel on the bottom right part of the figure. However in this case, most of the adjacent proteins of $320 \mathrm{~N}$ have low centrality values and might not be of interest.

As already discussed before, centrality values are usually used to identify the importance of proteins in a biological network. To find other important hubs in the network, the user may modify the range slider filters to hide all nodes with a small centrality value, by sliding a bar from the left to the right until only a small number of proteins remain for the selected centrality value. For our use-case, the betweenness centrality has been chosen as it relies on the overall network topology. High values of this centrality indicate central proteins in a PPI-network.

Figure 4(b) shows an example, where the purple colored Current Flow Betweenness (equivalent to the Random Walk Betweenness discussed in Section 2.2) has been adjusted. Protein $369 \mathrm{~N}$, which is the cellular tumor antigen p53, is selected here, as it has one of the highest betweenness values and most of the remaining visible nodes are adjacent to this protein and have high centrality values as well. Therefore, they could be important actors in the Mus musculus dataset. For instance, the adjacent protein $24169 \mathrm{~N}$, a tumor suppressor protein, might represent an important hub in the PPI-network and could be an interesting candidate for further investigation.

This small example shows an application of centrality measures in biological networks and how ViNCent can solve analysis problems by visualizing the networks interactively.

\subsection{Discussion}

Our visualization tool combines different approaches to overcome the difficulties when visualizing network centralities. It provides a new way of visualizing centrality values within a network by the use of a circular arrangement, and therefore, it minimizes visual clut- 


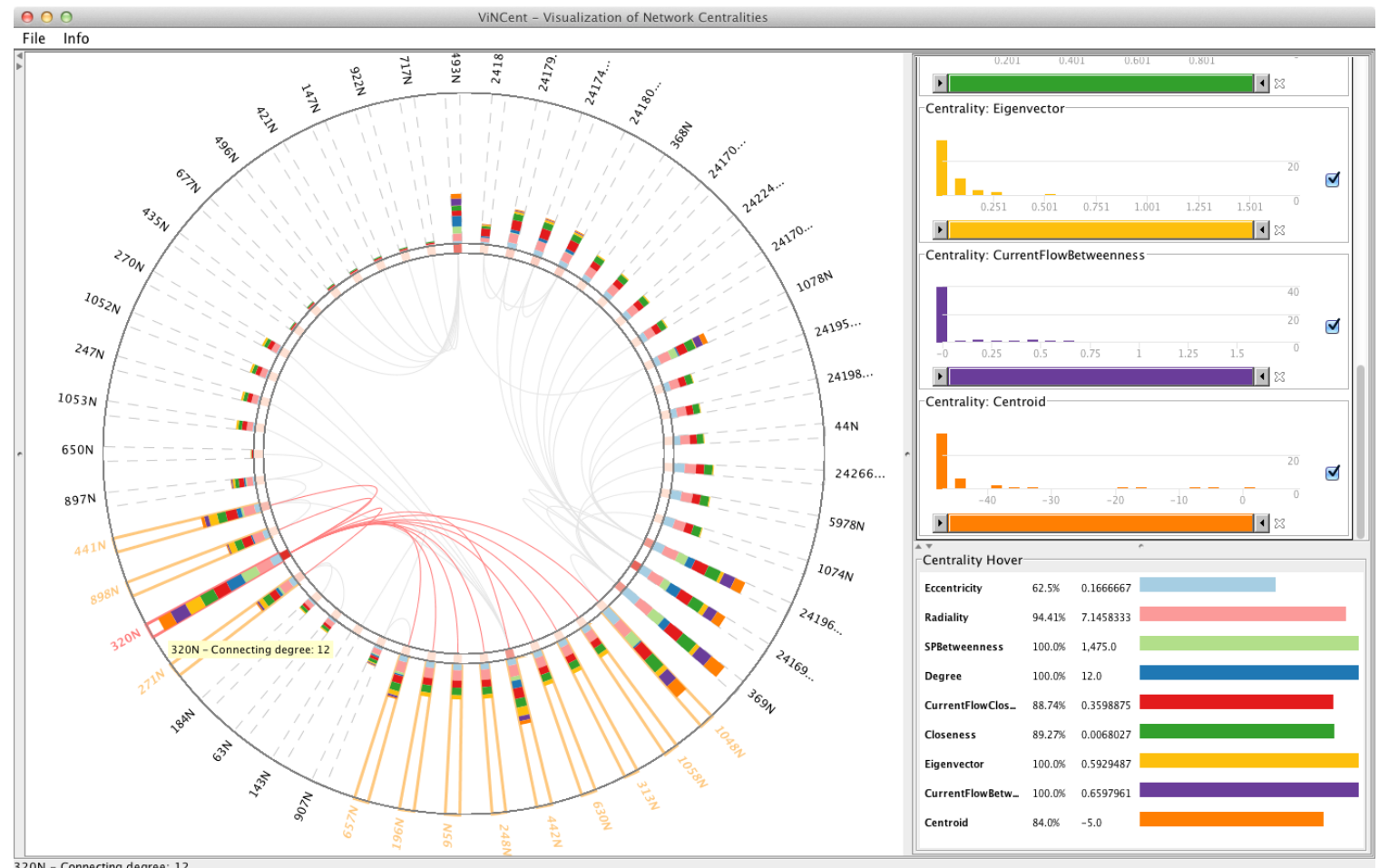

(a) Protein $320 \mathrm{~N}$ was selected for further consideration.

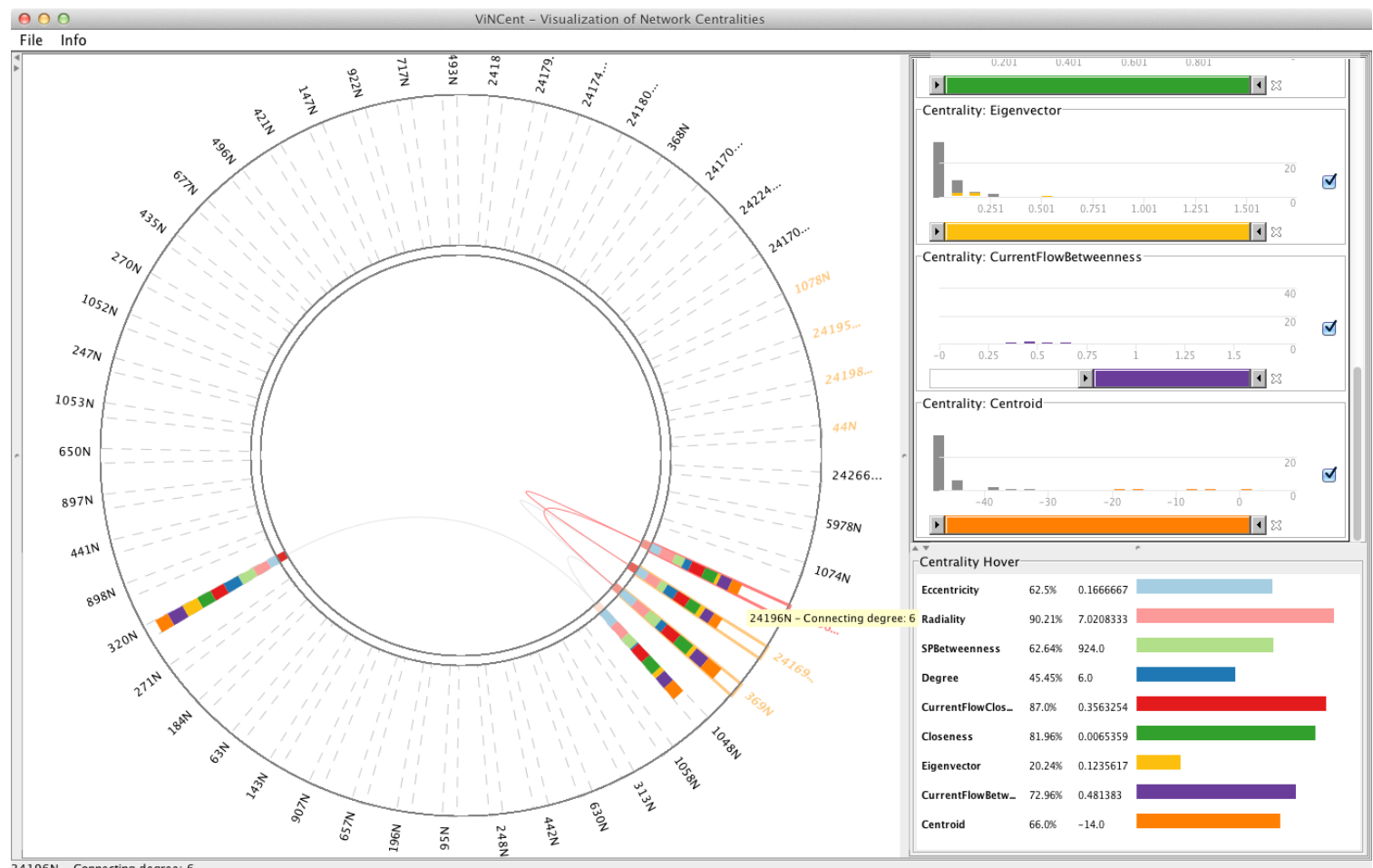

(b) Filtered view using histogram sliders of the ViNCent tool, showing the top proteins found by adjusting the CurrentFlowBetweenness. The first few bars of the other centralities are marked in dark gray, indicating that the corresponding nodes are already filtered out by the applied filter.

Figure 4: Use-case scenario of ViNCent. Filtering out important proteins in the Mus musculus dataset. 
ter and occlusions of nodes. The usage of stacked bars attached to the outside of the node representations gives the user the possibility to discover potentially important nodes (e.g., with high centrality values) at a first glance. The visual representation of the degree marking additionally supports this approach.

Another feature of ViNCent, which improves interactive exploration, is the way of filtering out data. By the use of histograms and thus the underlying distributions of network centrality values, the user can quickly get an overview and filter out unimportant data with a few clicks. The concepts of bar filtering, range sliders and single-node filtering allow the user to filter out any combination of nodes. This leads to less visual clutter in the drawing. We briefly summarize the advantages in the following and highlight the most important drawbacks:

Pros: Advantages are the circular arrangement of nodes, which leads to less visual clutter and allows the visualization of many nodes up to a few hundred. Other advantages are the filter possibilities that facilitate the exploration of networks and support the user in filtering out less important data at a first glance. The presented example shows how easy filters can be applied and how powerful they are when the user wants to reduce the amount of displayed data.

Cons: Current drawbacks are the lack of linking and brushing between histograms when hovering single bars (only already filtered out data is linked and brushed) and the missing visual links from the circle view to the histogram windows (so far, just hovering in histograms highlights nodes in the circle view). Especially the last issue would enhance the filter possibilities even more (e.g., hovering nodes in the circle view would show the location of the node in the histogram). Another drawback is the current performance: when dealing with a lot of data, the performance of the tool is not sufficient to maintain the full interaction possibilities. Linking and brushing features are then lacking of immediate feedback and lead to bad refreshing results for filtering tasks.

\section{CONCLUSION AND FUTURE WORK}

ViNCent can be used for any kind of network exploration and any kind of centrality visualization as long as the preconditions for the calculation of centrality values are met. Thus, it also supports a better understanding of multivariate networks. Compared to related tools, ViNCent performs well in visualizing centrality values for nodes, as it provides a direct vi- sual feedback by the use of different types of stacked bars. ViNCent still reveals the network structure and makes it therefore possible to follow paths in a network too. A number of interaction concepts support the mentioned features.

The tool scales well up to a few hundred nodes, depending on the available screen size and resolution. The size of the inner circle limits the number of nodes. However, there are features that are not implemented yet, such as the linking and brushing issue discussed in the previous section. In the following, we discuss possible future work by indicating further improvements.

An important future feature are new edge bundling modes, since they would lead to less visual clutter in the circle view. There is a lot of work for edge bundling in graphs, such as [Holten, 2006, Holten and van Wijk, 2009]. As some approaches do not rely on any further structure in the center of the circle view, they would be applicable to solve the edge bundling problem for ViNCent. As the computation of bundling edges is done in one single point so far, additional bundling modes could be introduced into the system.

Closely related to the problem of edge bundling is the arrangement of nodes on the circle itself. Depending on the node positions, edge bundling may produce better results. For this problem, one could take results of network analyses into account, like information concerning cliques or communities, single actors, or the density of the graph at certain positions. This information is useful to decide how nodes should be arranged along the circle, because based on this, we could calculate the possible amount of edge crossings.

So far, ViNCent uses the Prefuse toolkit [Heer et al., 2005] to render our visualizations. As this toolkit relies on Java2D instead of faster Java OpenGL implementations, the tool has some performance issues. Changing to a faster visualization toolkit may lead to better results.

Finally, the tool should be tested and evaluated with more complex biological networks and difficult tasks as well to clearly figure out problems of the chosen interaction and visualization techniques.

\section{ACKNOWLEDGEMENTS}

The authors wish to thank Ilir Jusufi, ISOVIS Group, Linnaeus University, as well as Falk Schreiber, Leibniz Institute of Plant Genetics and Crop Plant Research (IPK), Germany, for many constructive comments and their contributions to the system. 


\section{REFERENCES}

Albrecht, M., Kerren, A., Klein, K., Kohlbacher, O., Mutzel, P., Paul, W., Schreiber, F., and Wybrow, M. (2010). On open problems in biological network visualization. In Proc. International Symposium on Graph Drawing (GD '09), volume 5849 of LNCS, pages 256-267. Springer.

Brewer, C. A. (last accessed: 2011-03-22). ColorBrewer. http://colorbrewer2.org/, 2nd edition.

Correa, C. D., Crnovrsanin, T., Muelder, C., Shen, Z., Armstrong, R., Shearer, J., and Ma, K.-L. (2008). Cell phone mini challenge award: Intuitive social network graphs visual analytics of cell phone data using mobivis and ontovis. In Visual Analytics Science and Technology, 2008. VAST '08. IEEE Symposium on, pages $211-212$.

Correa, C. D. and Ma, K.-L. (2011). Visualizing social networks. In Aggarwal, C., editor, Social Network Data Analytics, pages 307-326. Springer.

Di Battista, G., Eades, P., Tamassia, R., and Tollis, I. G. (1999). Graph Drawing: Algorithms for the Visualization of Graphs. Prentice Hall.

Dwyer, T., Hong, S.-H., Koschützki, D., Schreiber, F., and $\mathrm{Xu}, \mathrm{K}$. (2006). Visual analysis of network centralities. In Misue, K., Sugiyama, K., and Tanaka, J., editors, Proceedings of the 2006 Asia-Pacific Symposium on Information Visualisation (APVis'06), pages 189-198, Darlinghurst, Australia. Australian Computer Society, ACM International Conference Proceeding Series, vol. 164.

Görg, C., Pohl, M., Qeli, E., and Xu, K. (2007). Visual Representations. In Kerren, A., Ebert, A., and Meyer, J., editors, Human-Centered Visualization Environments, LNCS Tutorial 4417, pages 163-230. Springer.

Heer, J., Card, S. K., and Landay, J. A. (2005). Prefuse: a toolkit for interactive information visualization. In Proceedings of the SIGCHI conference on Human factors in computing systems, CHI '05, pages 421-430, New York, NY, USA. ACM.

Henry, N., Fekete, J.-D., and Mcguffin, M. J. (2007). Nodetrix: a hybrid visualization of social networks. IEEE Transactions on Visualization and Computer Graphics (IEEE Visualization Conference and IEEE Conference on Information Visualization) Proceedings, 13:1302-1309.

Holten, D. (2006). Hierarchical edge bundles: Visualization of adjacency relations in hierarchical data. IEEE Transactions on Visualization and Computer Graphics, 12(5).

Holten, D. and van Wijk, J. J. (2009). Force-directed edge bundling for graph visualization. IEEE-VGTC Symposium on Visualization 2009, 28(3).

Jia, Y., Hoberock, J., Garland, M., and John C. Hart, Member, I.-C. (2008). On the visualization of social and other scale-free networks. IEEE Transactions on Visualization and Computer Graphics, 14(6):1285-1292.

Junker, B., Koschutzki, D., and Schreiber, F. (2006). Exploration of biological network centralities with centibin. BMC Bioinformatics, 7(1):219.

Junker, B. H. and Schreiber, F. (2008). Analysis of Biological Networks. Wiley Series on Bioinformatics, Computational Techniques and Engineering. Wiley.

Jusufi, I., Dingjie, Y., and Kerren, A. (2010). The network lens: Interactive exploration of multivariate networks using visual filtering. In Information Visualisation (IV), 2010 14th International Conference, pages 35 42.

Jusufi, I., Klukas, C., Kerren, A., and Schreiber, F. (2011) Guiding the interactive exploration of metabolic pathway interconnections. Information Visualization. (to appear).

Keim, D. A. (2002). Information visualization and visual data mining. IEEE Transaction on Visualization and Computer Graphics, 8(1):1-8.

Kerren, A. and Köstinger, H. (2011). Interactive exploration and analysis of network centralities. Interactive Poster, EuroVis 11, Bergen, Norway.

Koschützki, D. and Schreiber, F. (2004). Comparison of centralities for biological networks. In R. Giegerich, J. S., editor, Proc. German Conf. Bioinformatics (GCB04), pages 199-206.

Köstinger, H. (2011). Vincent - visualization of network centralities. Master's thesis, Linnaeus University, School of Computer Science, Physics and Mathematics, Växjö, Sweden.

Newman, M. E. J. (2003). A measure of betweenness centrality based on random walks. arXiv condmat/0309045.

Newman, M. E. J. (2010). Networks: An Introduction. Oxford University Press.

Roberts, J. C. (2007). State of the art: Coordinated \& multiple views in exploratory visualization. In $\mathrm{Pro}-$ ceedings of the Fifth International Conference on Coordinated and Multiple Views in Exploratory Visualization, pages 61-71, Washington, DC, USA. IEEE Computer Society.

Salwinski, L., Miller, C. S., Smith, A. J., Pettit, F. K., Bowie, J. U., and Eisenberg, D. (2004). The database of interacting proteins: 2004 update. Nucleic Acids Research, 32(1):449-451.

Scardoni, G., Petterlini, M., and Laudanna, C. (2009). Analyzing biological network parameters with centiscape. Bioinformatics, 25(21):2857-2859.

Shneiderman, B. (1994). Dynamic queries for visual information seeking. IEEE Software, 11:70-77.

Tweedie, L., Spence, B., Williams, D., and Bhogal, R. (1994). The attribute explorer. CHI'94 - Celebrating Interdependence, pages 435-436. 